\title{
COMMUNICATION
}

\section{Connaissances, attitudes et pratiques en pathologie de la muqueuse buccale des chirurgiens-dentistes en France, enquête nationale}

\author{
M. Roume (Paris), S. Azogui-Lévy (Paris), G. Lescaille (Paris), V. Descroix (Paris), J. \\ Rochefort (Paris)
}

Introduction : Les Chirurgiens-dentistes (CD) ont pour rôle d'identifier et de diagnostiquer les pathologies de la muqueuse orale (PMO), de les prendre en charge ou d'orienter les patients vers les spécialistes adéquats. Une importante errance diagnostique des patients atteints de PMO a été mise en évidence, pouvant être à l'origine d'une perte de chance notamment dans les cas de carcinomes épidermoïdes ou d'hémopathies malignes (Scully et al 1999, Albisetti et al, 2016). Ce constat s'accompagne d'études internationales portant sur le comportement des CD face aux PMO (Ergun et al 2009, Sardella et al, 2007), analysant les difficultés rencontrées et mettant en évidence un manque de connaissance que les praticiens attribuent à une formation universitaire insuffisante, mais également à un manque d'intérêt face aux PMO. Compte tenu de l'absence de données françaises, nous avons souhaité estimer la capacité des CD exerçant en France, à identifier, diagnostiquer et prendre en charge les PMO. Méthode - Nous avons réalisé en collaboration avec l'UFSBD (Union Française pour la Santé Bucco-Dentaire), une enquête nationale par questionnaire qui comprenait des items sur le diagnostic et la prise en charge des PMO. Sa diffusion s'est faite en ligne du 19 mai au 13 juillet 2017, 15000 adhérents ont été sollicités. Résultats - Le taux de réponses obtenu a été de $3.8 \%$, majoritairement des praticiens libéraux d'Ile de France, ayant en moyenne 20 ans d'exercice. Plus de $80 \%$ d'entre eux avaient participé à un congrès dans les 5 dernières années et $41 \%$ de ces formations concernaient les PMO. En revanche, les jeunes diplômés étaient moins nombreux à suivre ces formations. La quasi-totalité des praticiens (97\%) déclarait observer des lésions. Une majorité (65.5\%) effectuait un examen complet de la cavité orale lors de la 1èreconsultation, mais seulement $30 \%$ à chaque rendez-vous de suivi. Les CD déclaraient ne pas avoir de difficultés globales importantes à diagnostiquer et prendre en charge les lésions orales. Des différences significatives ont été notées selon les pathologies considérées et les caractéristiques du praticien : sexe, année de diplôme, mode d'exercice ou encore formations continues suivies ces 5 dernières années. La majorité des CD $(77 \%)$ expliquait leurs difficultés diagnostiques par un manque de patients présentant ce type de pathologies, 2/3 par un manque de confiance en leur diagnostic et leur connaissance sur ce thème et la moitié par une formation insuffisante. Conclusion - Notre enquête, a révélé des difficultés similaires à celles internationales : pas de réalisation systématique d'un examen oral complet, des difficultés élevées pour le diagnostic des carcinomes épidermoïdes, ce qui est inquiétant compte tenu du rôle des CD dans le dépistage des lésions. La mise en place de réseaux de soins facilitant la communication entre les différents acteurs de santé, spécialistes des lésions orales et praticiens libéraux, permettrait d'accélérer la prise en charge multidisciplinaire des patients et ainsi améliorerait le pronostic de certaines pathologies. 